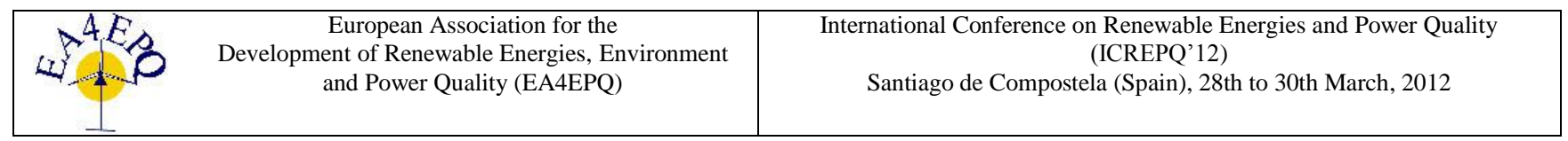

\title{
Development of a Monte Carlo simulation tool in order to minimize polluting gases emissions in presence of wind power under cost and load covering constraints
}

\author{
F. Vallée ${ }^{1}$, C. Versèle $^{2}$, J. Lobry $^{2}$ and F. Moiny ${ }^{1}$ \\ ${ }^{1}$ Department of General Physics \\ Faculté Polytechnique, University of Mons \\ Rue de Houdain 9, 7000 Mons (Belgium) \\ Phone number: +0032 65 374043, e-mail: francois.vallee@ umons.ac.be, francis.moiny@umons.ac.be \\ ${ }^{2}$ Department of Electrical Power Engineering \\ Faculté Polytechnique, University of Mons \\ Boulevard Dolez 31, 7000 Mons (Belgium) \\ Phone: +0032 65 374186, e-mail: Christophe.versele@umons.ac.be, Jacques.lobry@umons.ac.be
}

\begin{abstract}
In this paper, an original non sequential Monte Carlo simulation tool is developed. This tool permits to compute the optimal dispatch of classical (coal, oil,...) thermal generation in order to minimize polluting gases $\left(\mathrm{NO}_{\mathrm{x}}, \mathrm{CO}_{2}, \ldots\right)$ emissions in presence of wind power and under constraints like the maximal generation cost or the ability of the electrical system to cover the load. In comparison with existing analytical tools based on restrictive hypotheses when it comes to wind power modelling (generally represented by a single entirely correlated global wind park), unexpected outages of conventional parks or fluctuating representation of the load, the use of Monte Carlo simulation allows to remove all those limitations. Indeed, thanks to the developed tool, the optimal dispatch of classical thermal generation can be reached under several load conditions. Well-known reliability indices can also be computed and moreover, following the wind speed sampling that is used, entirely correlated, independent or more accurate correlation level between wind parks can be considered. Finally, it is thought that the proposed solution can be a useful tool for electrical system operators in order to dispatch the polluting thermal units under cost, reliability, emissions, fluctuating wind power and unexpected outages constraints.
\end{abstract}

\section{Key words}

Gaseous pollutants emissions, Monte Carlo simulation, wind generation, electrical system management, technical analysis.

\section{Introduction}

Nowadays, most of the conventional electrical parks are still using fossil resources like coal or oil. Those primary resources involve the emission of gaseous pollutants such as carbon oxides $\left(\mathrm{CO}_{\mathrm{x}}\right)$ or oxides of nitrogen $\left(\mathrm{NO}_{\mathrm{x}}\right)$. Recently, following the Kyoto agreements, a great research effort has been made in order to reduce those emissions worldwide. In this context, one of the most promising alternative resources is certainly wind power. Given the fluctuating behaviour of the wind and due to several operating constraints (cost, reliability,...) related to electrical systems, it is important to adequately dispatch conventional thermal generation in order to guarantee a minimization of the gaseous pollutants emissions and to simultaneously face the requirements of modern networks. In that way, references [1] and [2] have proposed analytical models in order to minimize emissions under cost and load covering constraints. However, even if they represent a first approach, those models are limited by several hypotheses. For example, it can be quoted that the hourly load fluctuations cannot be analytically taken into account and that the computations are therefore made for a single load value. Moreover, investigated correlation scenarios between wind parks are relatively limited with the analytical approach and wind generation is generally supposed to be entirely correlated over the studied territory. Finally, as only one single load value is considered, it is not possible to compute reliability indices like the Loss of Load Probability (LOLP) or the Loss of Load Expectation (LOLE) [3]-[4] with the existing analytical tools. In order to avoid the drawbacks of the analytical approach, it is therefore proposed in this paper to develop a non sequential Monte Carlo simulation tool [5]-[6] that permits to consider a load fluctuating behaviour but also several correlation scenarios between wind parks. Thanks to the developed tool, an optimal dispatch of the classical thermal generation can be realized for each generated system state, reliability indices can be evaluated and wind generation long term impact on gaseous pollutants emissions can be studied. Concerning this last point, it is important to note that the simulated states are independent steady-state ones and that, therefore, short term transitions between system states cannot be studied 
here. By consequence, the eventual increase of $\mathrm{CO}_{2}$ emissions due to the fast starting of coal thermal units in case of unexpected lack of wind is beyond the scope of the present study. Nevertheless, in order to prove the utility of the developed simulation tool for the system operators from the long term electrical dispatch point of view, it has been applied to a slightly modified version of the Roy Billinton Test System (RBTS) [7].

Finally, the present paper is organized as follows. In a first section, the general algorithm of the developed Monte Carlo simulation tool is presented. Then, the optimization model solved during each generated state of the simulation is described. In a third part, some simulation results and applications of the developed tool are proposed in order to show its usefulness. Finally, a conclusion pointing out the main interests of the present work is proposed.

\section{General algorithm of the developed Monte Carlo simulation tool}

Monte Carlo simulations are generally used to simulate the actual process and random behaviour of a given electrical system. The pursued objectives are, for example, the computation of reliability indices, the search for profitable investments scenarios, and so on [4], [6]... Theoretically, there are two basic techniques used when Monte Carlo methods are considered for power system applications, these methods being known as the sequential and non sequential techniques [6], [8]. In that way, in the present study, a non sequential Monte Carlo algorithm (figure 1) has been developed under Matlab ${ }^{\circledR}$ to evaluate reliability indices of interest, gaseous pollutants mean emissions, adequate dispatch of classical units,... This algorithm is only limited to hierarchical level HLI [4]. It means that the total system generation is examined with the total system load requirement on a pooled basis. Moreover, the transmission system and its ability to move the generated energy towards the consumer is ignored in a HLI study.

The developed Monte Carlo simulation could theoretically incorporate any number of system parameters and states but it has been assumed in our calculation that a classical generation unit was only able to reside in one of the following two states: fully available and unavailable. Moreover, in the established non sequential simulation, only hourly uncorrelated states are considered as it is supposed that a generation unit outage state does not condition or influence its state during the next or previous hours of simulation (and inversely). Consequently, at the start of each hour, a uniformly distributed random number $(u)$ on the interval $[0,1]$ is sampled for each classical (thermal, hydroelectric,...) generation unit in order to decide its operation state, using the following process:

$\checkmark \quad$ If $u \leq$ Forced Outage Rate (FOR) [4], the classical unit is decided to be unavailable;

$\checkmark \quad$ If $\mathrm{u}>\mathrm{FOR}$, the classical unit is decided to be fully available;

Concerning the hourly load of the system, its determination in a non sequential algorithm can be practically based on a random sampling over its cumulative distribution function [5] or, more precisely, established by the use of modulation diagrams of the annual peak load value [9]:

$\checkmark$ Diagram of weekly modulation of the annual peak load: this last one permits to calculate the peak load of the current week on the basis of the annual peak load value. This diagram contains thus 52 modulation rates of the annual peak load value;

$\checkmark$ Diagram of the hourly modulation of the weekly peak load: it permits to calculate the hourly load for each hour of the week. This diagram contains thus 24 modulation rates of the weekly peak load value.

Using this last methodology, no random sampling is needed in order to generate the hourly load of the system. More easily, the program just considers, in the weekly modulation diagram, the rate corresponding to the current week during the simulation process (figure 1). Then, it associates to the generated weekly peak load the rate of the hourly modulation diagram corresponding to the investigated hour of the day. Also note that, as the consumption during one week can change from one day to the other (days of the week, Saturday or Sunday), several diagrams of hourly modulation can be defined during one week. Moreover, seasonal aspects can also be taken into account by defining periods during the year and by changing the set of hourly modulation diagrams associated to the load from one period to the other.

From the wind generation point of view, several modeling methods like the ones based on AutoRegressive Moving Average models have already been proposed in the literature and require increased computing capacities [10]. Here, as we consider a non sequential approach, it is not necessary to establish such a complex chronological model for wind speeds but it will be more efficient to introduce a random sampling based on a defined statistical distribution [5], [9] associated to each wind park. Practically, the non sequential wind speed sampling is generally based on a direct inversion of the Cumulative Distribution Function [5], [9]. More precisely, the process can be described as follows:

$\checkmark \quad$ Sampling of an uniformly distributed number ' $\mathrm{u}$ ' on the interval $[0,1]$;

$\checkmark$ Application of that sampled random number ' $u$ ' to the Cumulative Distribution Function in order to determine the associated wind speed.

Given the expected wind correlation scenario, the same random number ' $u$ ' can be sampled for all the wind parks. In that case, they are all supposed to be entirely correlated. At the opposite, the parks can be entirely independent by sampling an independent random number ' $u$ ' for each of them. Otherwise, more accurate correlation scenarios can be reached by combining for each wind park an adequately computed noise with a single mean distribution [11]. Finally, note that a power curve is associated to each wind park and permits to 
convert the sampled wind speed into generated power.

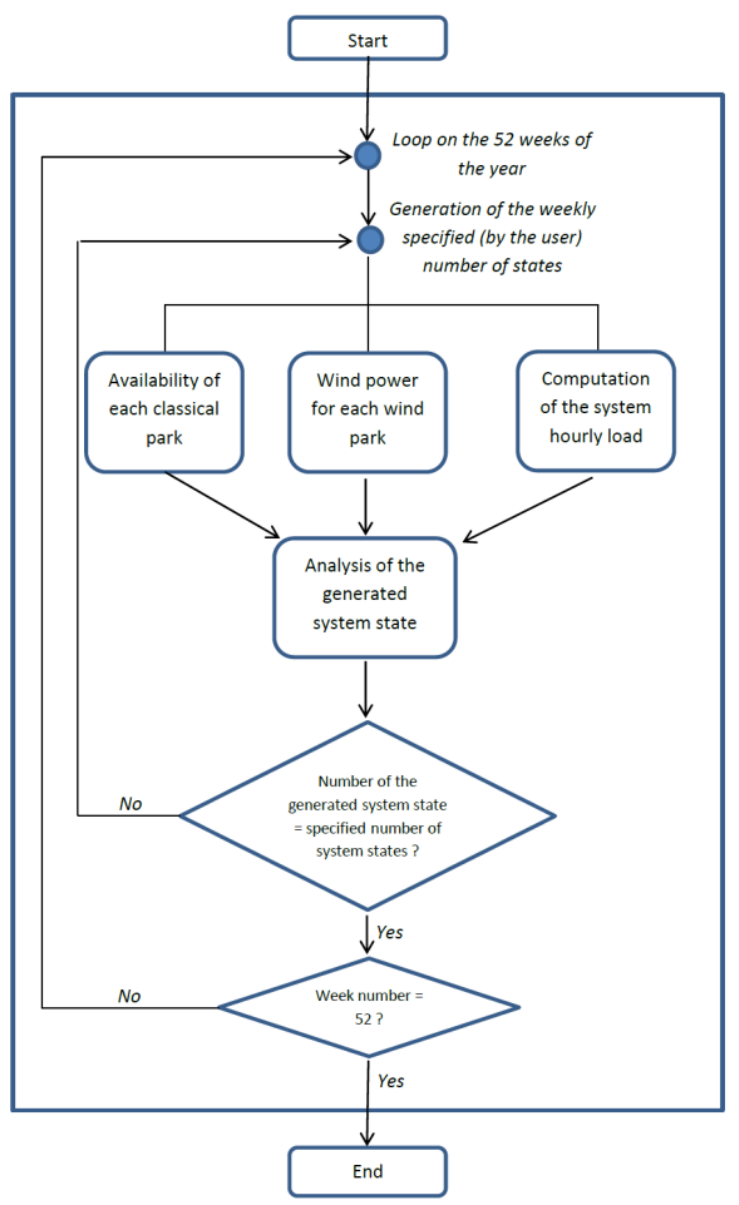

Fig.1. Algorithm of the developed Monte Carlo simulation tool

At the end of the system states generation process, reliability indices like the LOLP and the LOLE [3]-[4] can be computed based on the simulated states during which the hourly load cannot be fully covered with the available (classical + wind) generation.

Moreover, during the Monte Carlo process, each generated system state must be analyzed in order to minimize (under cost and load covering constraints) the gaseous pollutants emissions by adequately dispatching the available (classical + wind) generation. In that way, the implemented optimization model is presented in section 3 .

\section{Optimization model to be solved during each generated system state}

Before presenting the optimization model developed in this section, it is important to note that only coal and oil thermal units, hydroelectric generation and wind parks are considered in this paper. Nevertheless, the proposed model could be easily extended to other kinds of production means like nuclear units

The optimization model presented in this section tends to minimize $\mathrm{NO}_{\mathrm{x}}$ emissions under cost and load covering constraints. In that way, the pursued objective is based on the definition of an Environmental Impact Index (EII) expressed in tons per hour. The contribution of classical thermal (coal or oil) units to this index can be defined like in references [1] and [2]:

$$
E I I=a_{0}+a_{1} \cdot x_{t}+a_{2} \cdot x_{t}^{2}+a_{3} \cdot \exp \left(a_{4} \cdot x_{t}\right)
$$

where, $x_{t}$ is the generated power (expressed in per unit) of the considered classical thermal (oil, coal,...) unit and $\mathrm{a}_{\mathrm{i}}(\mathrm{i}=1, \ldots, 4)$ are empirical coefficients related to this unit. Note that one of the main characteristics of the EII index comes from its U-shape in function of the generated thermal power.

Finally, note that hydroelectric and wind generation units can be approximated as zero emission units [12] and do therefore not have any contribution to the EII.

From the cost $\mathrm{C}(\$ / \mathrm{h})$ point of view, it is based on a strictly growing evolution in function of the generated power [13]. In that way, the cost associated to classical thermal units $C_{t}(\$ / h)$ is generally characterized by a fuel cost square function of the generated power [13] and a starting investment cost quite limited in comparison with large hydroelectric units [14]:

$$
C_{t}=q_{t 0}+q_{t 1} \cdot x_{t}+q_{t 2} \cdot x_{t}^{2}
$$

where, $x_{t}$ is again the generated power (expressed in per unit) of the thermal unit and $\mathrm{q}_{\mathrm{ti}}$ are empirical coefficients related to this unit.

Concerning the hydroelectric parks cost $C_{h}(\$ / h)$, as already mentioned, the starting investment cost is generally quite high and the operating cost is quite reduced (as the fuel cost is zero). The latter is mainly due to maintenance activities and is practically modelled by a linear evolution depending of the generated power [14]:

$$
C_{h}=q_{h 0}+q_{h 1} \cdot x_{h}
$$

where, $x_{h}$ is the generated power (expressed in per unit) of the hydroelectric unit and $\mathrm{q}_{\mathrm{hi}}$ are empirical coefficients related to this unit.

From the wind power point of view, given the multiple encouraging governmental policies in many countries, it has to be considered as a must run zero cost $\left(\mathrm{C}_{\mathrm{w}}=0\right)$ generation unit in the proposed tool. Indeed, thanks to this hypothesis, wind power will be considered before classical units in order to cover the load and will be in agreement with the reality [15].

Practically, in order to express the cost constraint, a maximal hourly cost is defined [1]-[2] and the constraint is defined as:

$$
\sum_{i=1}^{n_{h}} C_{h i}+\sum_{i=1}^{n_{t}} C_{t i}+\sum_{i=1}^{n_{w}} C_{w i} \leq C_{\max }
$$

where, $n_{w}, n_{t}$ and $n_{h}$ are respectively the number of installed wind, thermal and hydroelectric units. $\mathrm{C}_{\max }$ is the maximal cost fixed for the hourly electrical generation.

The load constraint associated to the optimization problem consists in exactly covering the load with the optimally dispatched classical and wind generation units. This constraint can thus be written as: 


$$
\sum_{i=1}^{n_{w}} P_{o p t w i}+\sum_{i=1}^{n_{t}} P_{o p t t i}+\sum_{i=1}^{n_{h}} P_{o p t h i}=P_{d}
$$

where, $\mathrm{P}_{\text {optwi }}, \mathrm{P}_{\text {optti }}$ and $\mathrm{P}_{\text {opthi }}$ are respectively the hourly optimal generation of wind, thermal and hydroelectric units. In the same way, $P_{d}$ represents the hourly load demand.

Using expressions (1), (4) and (5), the minimization model of $\mathrm{NO}_{\mathrm{x}}$ emissions under cost and load covering constraints can be expressed as:

$$
\begin{aligned}
& \operatorname{Min} E I I=\sum_{i=1}^{n_{t}}\left[a_{i 0}+a_{i 1} \cdot x_{t i}+a_{i 2} \cdot x_{t i}^{2}+a_{i 3} \cdot \exp \left(a_{i 4} \cdot x_{t i}\right)\right](6) \\
& \sum_{i=1}^{n_{h}} C_{h i}+\sum_{i=1}^{n_{t}} C_{t i}+\sum_{i=1}^{n_{w}} C_{w i} \leq C_{\max } \\
& \sum_{i=1}^{n_{w}} P_{\text {optwi }}+\sum_{i=1}^{n_{t}} P_{\text {optti }}+\sum_{i=1}^{n_{h}} P_{\text {opthi }}=P_{d}
\end{aligned}
$$

For efficiency and security reasons, each classical unit must also be constrained by lower $\left(\mathrm{x}_{\min , \mathrm{i}}\right)$ and upper $\left(\mathrm{x}_{\max , \mathrm{i}}\right)$ boundary values:

$x_{\min , i} \leq x_{i} \leq x_{\max , i} \quad\left(\mathrm{i}=1,2, \ldots, \mathrm{n}_{\mathrm{c}}\right)$

where, $n_{c}$ is the total number of classical units $\left(n_{c}=n_{t}+\right.$ $\mathrm{n}_{\mathrm{h}}$ ).

The developed optimization model is thus characterized by an objective function and constraints derivable and continuous in each point. Consequently, the optimal solution associated to each generated system state is calculated by use of the fmincon solver from the Matlab ${ }^{\circledR}$ Optimization Toolbox. This optimizer provides local optimization based on a gradient descent algorithm and can thus be limited in the case of problems with multiple local optima. However, the objective function (6) is always characterized by a single optimum and is thus perfectly suited for the application of the fmincon solver. Finally, note that this solver can also handle several types of constraints (linear or non-linear, equality, inequality,...).

\section{Calculated indices and useful results provided by the Monte Carlo simulation tool}

\section{A. Calculated reliability indices}

In the Monte Carlo Simulation tool, the power system is thus modeled by specifying a set of 'events', where an event is a random occurrence that changes the system state. In the present study, the events recognized by the established program are the changes in load, the possible failure of a generating unit or the variation of the power produced by a wind park. Each simulated system state is then defined in terms of available margin, which is the difference between the available (classical + wind) generation capacity and the load. In order to proceed to this step, the total available system capacity must be superimposed on the load during each simulated hour to define:

$\checkmark$ Healthy state: The total available capacity is greater than the corresponding hourly load;

$\checkmark$ Risky state: The total available capacity is less than the corresponding hourly load value.

Two complementary indices among which the LOLP [3][4] are then defined as:

Probability of health $=P(H)=\frac{n(H)}{N \times 8760}$
Probability of risk $(L O L P)=P(R)=\frac{n(R)}{N \times 8760}$

where, $n(H)$ and $n(R)$ are respectively the total number of hourly healthy and risky simulated states; $\mathrm{N}$ being the total amount of simulated years.

The number of hours per year during which the available total (classical + wind) generation cannot meet the load is defined as the Loss of Load Expectation (LOLE) index and is obtained by multiplying the LOLP index with the annual number of hours (8760 hours per year).

\section{B. Optimization results}

Risky states cannot be optimized as the available generation is lower than the load. Consequently, the load constraint (8) cannot be met during those states. An indicator of risky states has thus been defined and set to 1 when such a state is simulated. Moreover, during a risky state, all the available generation has to operate at its maximal point in order to face the lack of production.

During healthy states, the previous indicator is set to 0 and it is worth to solve the optimization problem. In that way, in some healthy cases, the cost constraint cannot be met with the available generation and the load can thus not be fully covered with an adequate cost. Such cases are also characterized by another indicator set to 1 when the maximal cost constraint is violated. Healthy states presenting an optimized solution that verifies both load and cost constraints have both indicators set to 0 . Moreover, thanks to the Monte Carlo environment, a focus can be made on the optimal configuration of each of those healthy states. This property of the developed tool could be useful for the electrical system operator in order to adequately dispatch the generation park when gaseous pollutants emissions are taken into account. Finally, at the end of the simulation, a mean EII index can be computed and permits, for example, to quantify the impact of wind power on the reduction of gaseous pollutants emissions.

\section{Simulation results on a modified RBTS test system}

In order to test the developed Monte Carlo simulation tool, a slightly modified version of the academic RBTS test system has been considered. In its initial version [7], the RBTS was consisting of 7 hydroelectric units and 4 classical thermal parks. Unfortunately, wind generation 
was not taken into account in that version of the RBTS. In order to introduce that renewable energy into the Roy Billinton Test System, reference [16] has consequently proposed the addition of wind power based on Weibull statistical distributions in the context of HLI adequacy studies. In the present paper, two wind parks with an installed capacity of 2 p.u., each, are considered. Both are subject to Weibull distributions with scale (A) and shape (B) parameters being respectively $(\mathrm{A}=10.81 ; \mathrm{B}=1.41)$ and $(\mathrm{A}=11.25 ; \mathrm{B}=1.20)$ [17]. Wind power is finally obtained by use of a variable speed classical power curve for each park [18]. In order to keep a sufficient number of simulated risky states and to test our simulation tool in every condition, the annual peak load value of the RBTS has been increased by 3.5 p.u. (annual peak load value = 22 p.u.) jointly to the introduction of wind generation. This new load value is afterwards modulated by use of the Belgian hourly and weekly modulation rates [9]. Finally, the empirical emission coefficients $a_{i j}(j=0, \ldots, 4)$ associated to each thermal unit ' $\mathrm{i}$ ' are extracted from references [1]-[2] and are listed in table 1.

Table 1 - Empirical emission coefficients associated to

\begin{tabular}{|c|c|c|c|c|c|}
\multicolumn{7}{c|}{ classical thermal units } \\
\hline & $\mathrm{a}_{\mathrm{i} 0}$ & $\mathrm{a}_{\mathrm{i} 1}$ & $\mathrm{a}_{\mathrm{i} 2}$ & $\mathrm{a}_{\mathrm{i} 3}$ & $\mathrm{a}_{\mathrm{i} 4}$ \\
\hline $\begin{array}{c}\text { Thermal } \\
\text { unit 1 }\end{array}$ & 0.04091 & -0.05554 & 0.0649 & 0.0002 & 2.857 \\
\hline $\begin{array}{c}\text { Thermal } \\
\text { unit 2 }\end{array}$ & 0.02543 & -0.06047 & 0.05638 & 0.0005 & 3.333 \\
\hline $\begin{array}{c}\text { Thermal } \\
\text { unit 3 }\end{array}$ & 0.04258 & -0.05094 & 0.04586 & 0.000001 & 2.857 \\
\hline $\begin{array}{c}\text { Thermal } \\
\text { unit 4 }\end{array}$ & 0.05326 & -0.0355 & 0.0338 & 0.002 & 2 \\
\hline
\end{tabular}

In the same way, the empirical cost coefficients associated to thermal and hydroelectric parks are taken from references [1], [2] and [14] and are respectively summarized in tables 2 and 3 :

Table 2 - Empirical cost coefficients associated to classical thermal units

\begin{tabular}{|c|c|c|c|}
\hline & $\mathrm{q}_{\mathrm{t} 0}$ & $\mathrm{q}_{\mathrm{t} 1}$ & $\mathrm{q}_{\mathrm{t} 2}$ \\
\hline Thermal unit 1 & 10 & 200 & 100 \\
\hline Thermal unit 2 & 10 & 150 & 120 \\
\hline Thermal unit 3 & 20 & 180 & 40 \\
\hline Thermal unit 4 & 10 & 100 & 60 \\
\hline
\end{tabular}

Table 3 - Empirical cost coefficients associated to hydroelectric

\begin{tabular}{|c|c|c|}
\multicolumn{1}{|c}{ units } \\
\hline Hydrolectric 1 & $\mathrm{q}_{\mathrm{h} 0}$ & $\mathrm{q}_{\mathrm{h} 1}$ \\
\hline Hydrolectric 2 & 30 & 50 \\
\hline Hydrolectric 3 & 30 & 65 \\
\hline Hydrolectric 4 & 35 & 35 \\
\hline Hydrolectric 5 & 25 & 40 \\
\hline Hydrolectric 6 & 25 & 50 \\
\hline Hydrolectric 7 & 20 & 60 \\
\hline
\end{tabular}

Finally, note that the installed classical (thermal + hydroelectric) generation capacity is reaching 24 p.u. in our development and that the number of simulated system states is defined such as a $10^{-4}$ accuracy can be reached on the computed indices.

\section{A. Results collected with the developed simulation tool for the modified RBTS with independent wind parks}

In a first simulation, both wind parks are supposed to be entirely independent and the obtained results are shown for one week in figures 2 .a and b, 3 and 4 .

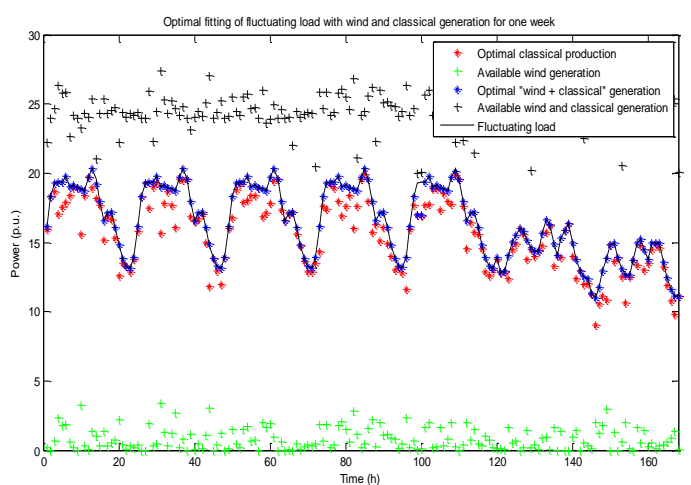

(a)

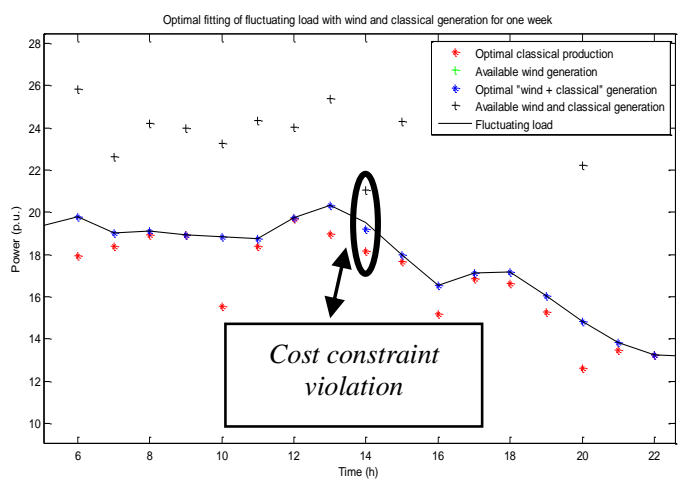

(b)

Fig.2. Optimal fitting of the fluctuating load with wind and classical generation for one week (a) ; zoom on one day (b)

It can be clearly observed in figure 2 that the optimal solution from the gaseous pollutants emissions point of view adequatly follows the load. However, in some cases like the one that can be pointed out during hour 14, the available «classical + wind » generation is greater than the load but the optimally dispatched « classical + wind » generation stays below the load. This result can be explained by looking at figure 3 . Indeed, it is due to the fact that the cost constraint $\left(\mathrm{C}_{\max }=3000 \$ / \mathrm{h}\right)$ cannot be faced with the available generation and the load that has to be covered.

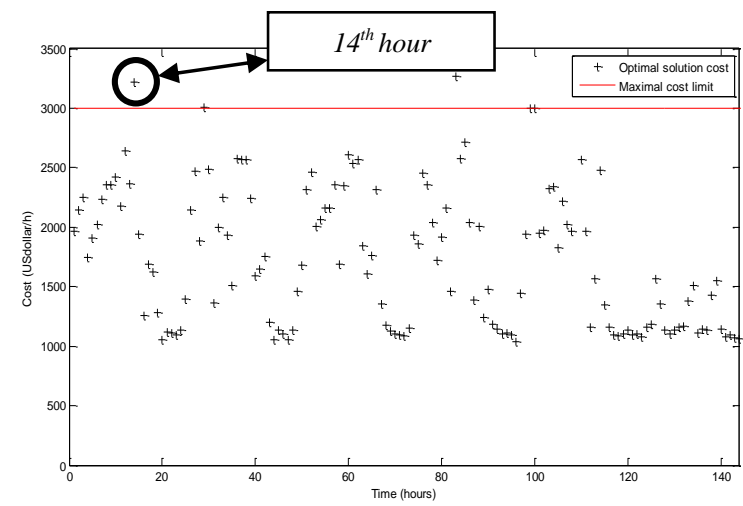

Fig.3. Cost of the optimal solution for each simulated state (cross) and maximal cost constraint (continuous) 
The indicator of loss of load states and the hourly EII indices can also be plotted thanks to the developped tool. Both indicators are proposed in figure 4 and it can be easily concluded that no LOLP states are to be seen during the considered week (LOLP indicator always equal to 0 ).

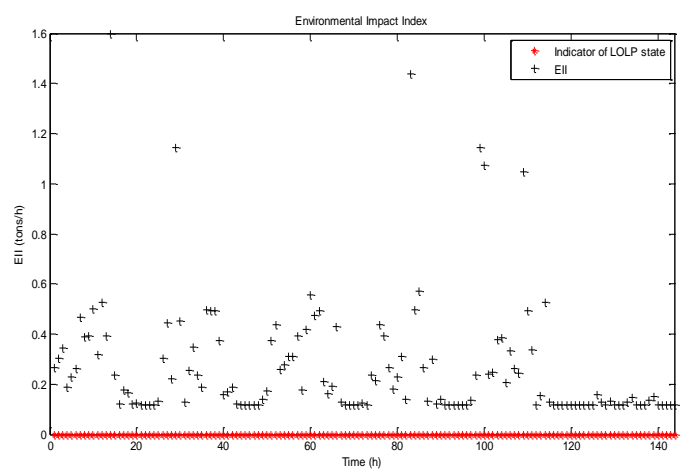

Fig.4. Environmental Impact Index (black cross) and LOLP state indicator (red star) during one simulated week

Finally, at the end of the simulation, the LOLP, LOLE (in hours/years) and mean EII (in tons/hour) are computed by the software and are displayed on the Matlab® workspace.

\section{B. Impact of the wind generation on the collected reliability (LOLP, LOLE) and emission (mean EII) indices}

Thanks to the developed simulation tool, it is possible to quantify the impact of wind generation on the electrical system adequacy and on gaseous pollutants emissions. In that way, the same wind parks as the ones defined in the introduction of section 5 have been considered and the installed wind capacity has been increased from 4 p.u. ( 2 p.u. at each wind park) to 8 p.u. (4 p.u. at each wind park). Moreover, in order to also quantify the impact of wind correlation on the obtained simulation results, two extreme scenarios have been computed: on the one side, both wind parks have been supposed to be entirely correlated and, on the other side, the same wind parks have been considered as entirely independent. Finally, note that all the simulations have been realized with the same unchanged load and classical generation characteristics as the ones detailed for the modified RBTS.

Table 4. Impact of wind correlation and installed capacity on the electrical system adequacy and gaseous pollutants emissions (E C = Entirely correlated ; I = Independent $)$

\begin{tabular}{|c|c|c|c|c|c|c|}
\hline $\begin{array}{c}\text { Installed } \\
\text { wind } \\
\text { capacity } \\
\text { (p.u.) }\end{array}$ & \multicolumn{2}{|c|}{ LOLP } & \multicolumn{2}{c|}{ LOLE (h/year) } & \multicolumn{2}{c|}{$\begin{array}{c}\text { Mean EII } \\
\text { (tons/year) }\end{array}$} \\
\hline & E C & I & E C & I & E C & I \\
\hline 4 & 0.0026 & 0.0021 & 22.93 & 17.95 & 0.2012 & 0.1959 \\
\hline 6 & 0.0022 & 0.0015 & 19.44 & 13.14 & 0.1939 & 0.1843 \\
\hline 8 & 0.0017 & 0.0013 & 14.95 & 10.97 & 0.1834 & 0.1745 \\
\hline
\end{tabular}

Based on table 4, it can be easily observed that the increase of wind penetration (entirely correlated or not) is improving both emissions and reliability indices. This result is quite logical as the load and classical generation characteristics are not changed while the installed wind capacity is increased. Therefore, as wind generation is considered as a zero cost non polluting generation mean, it will be preferentially used to cover the load and will consequently decrease the gaseous pollutants emissions. Moreover, as the load is not changed, the increase of wind penetration increases also the installed total (wind + classical) generation capacity and tends thus to decrease the risk of facing states of load non recovering.

On the other side, when the correlation between wind parks is analyzed, it can be seen that the power smoothing implied by the independence scenario between wind parks (an important production for one wind park does not necessarily involve a consequent production for the other as the parks are supposed to be independent) tends to reduce the risk of load non recovering and leads to reduced gaseous pollutants emissions in comparison with the "entire correlation" scenario. Indeed, when wind parks are entirely correlated, the simulated wind power is much more fluctuant. Therefore, in some states during which the available wind generation is very law (for all the wind parks due to the entire correlation between them), the risk of not being able to cover the load is increased and much more classical thermal generation is needed with, as a consequence, an increase of the gaseous pollutants emissions.

\section{Conclusions}

In this paper, an original Monte Carlo simulation tool has been developed in order to optimally dispatch, from the gaseous pollutants emissions point of view, thermal generation under load covering and cost constraints. With the developed tool, the random behaviour of wind generation and of the hourly load can also be taken into account and represents therefore a consequent improvement in comparison with the quite limited existing analytical approach. Moreover, thanks to the proposed software, the long term impact of wind generation on the electrical system adequacy and on gaseous pollutants emissions can be evaluated in different wind correlation scenarios and allows thus to better quantify the real influence of wind generation. Finally, it is thought that the developed tool could be a great help for electrical system operators in order to adequately dispatch the available classical thermal generation in presence of wind power and under reliability, cost and minimized gaseous pollutants emissions constraints.

\section{Bibliography}

[1] X. Liu, W. Xu, "Minimization emission dispatch constrained by stochastic wind power availability and cost", IEEE Trans. on Power Systems, vol. 25, n`3, pp. 1705-1713, Aug. 2010.

[2] X. Liu, "Emission minimisation dispatch constrained by cost and wind power", IET Gener. Transm. Distrib., vol. 5, n7, pp. 735742, July 2011.

[3] J.F. Manwell, J.G. Mc Gowan, A.L. Rogers, "Wind energy explained: Theory, design and application", $2^{\text {nd }}$ edition, Wiley, chap.11, pp. 24-25, 2009.

[4] R. Billinton, R.N. Allan, "Reliability evaluation of power systems", $2^{\text {nd }}$ edition, Springer, chap.1\&2, 1996. 
[5] G. Papaefthymiou, P.H. Schavemaker, L. van der Sluis, W.L. Kling, D. Kurowicka, R.M. Cooke, "Integration of stochastic generation in power systems", Electrical Power and Energy Systems, Elsevier, vol. 28, n 9, pp. 655-667, 2006.

[6] R. Billinton, R. Karki, "Application of Monte Carlo simulation to generating system well-being analysis", IEEE Trans. on Power Systems, vol. 14, n³, pp. 1172-1177, Aug. 1999.

[7] R. Billinton, S. Kumar, N. Chowdhury, K. Chu, K. Debnath, L. Goel, E. Khan, P. Kos, G. Nourbakhsh, J. Oteng-Adjei, "A reliability test system for educational purposes - Basic data”, IEEE Trans. on Power Systems, vol. 4, n³, pp. 1238-1244, Aug. 1989.

[8] W. Wangdee, R. Billinton, "Bulk electric system well-being analysis using sequential Monte Carlo simulation", IEEE Trans. on Power Systems, vol. 21, n¹, pp. 188-193, Feb. 2006.

[9] F. Vallée, J. Lobry, O. Deblecker, "Wind generation modelling to help the managerial process of modern transmission systems", Renewable Energy, Elsevier, vol. 36, pp. 1632-1638, May 2011.

[10] W. Wangdee, R. Billinton, "Considering load-carrying capability and wind speed correlation of WECS in generation adequacy assessment", IEEE Trans. on Energy Conversion, vol. 21, n³, pp. 734-741, Sept. 2006.

[11]F. Vallée, J. Lobry, O. Deblecker, "Application and comparison of wind speed sampling methods for wind generation in reliability studies using non sequential Monte Carlo simulations", European Transactions on Electrical Power, Wiley, vol. 19, pp. 1002-1015, 2009.

[12]Department of Energy and Environmental Protection Agency, "Carbon dioxide emissions from the generation of electric power in the United States", working group report, pp. 2-3, July 2000.

[13] J.H. Talaq, F. El-Hawary, M. E. El-Hawary, "Minimum emissions power flow”, IEEE Trans. on Power Systems, vol. 9, n¹, pp. 429435, Feb. 1994.

[14]J.M. Martin-Amouroux, "The economics of hydroelectricity", Hydro21, Grenoble European Center on Hydropower, pp. 1-4, June 2004.

[15] R. Mackensen, B. Lange, F. Schlögl, "Integrating wind energy into public power supply systems - German state of the art", International Journal of Distributed Energy Sources, vol. 3, $\mathrm{n}^{\circ} 4$, pp. 259-271, 2007.

[16]B. Raison, M. Crappe, J. Trecat, "Effets de la production décentralisée dans les réseaux électriques”, Sous projet 5, FPMs, Sept. 2001.

[17]F. Vallée, G. Brunieau, M. Pirlot, O. Deblecker, J. Lobry, "Optimal wind clustering methodology for adequacy evaluation in system generation studies using non sequential Monte Carlo simulation", IEEE Trans. on Power Systems, vol. 26, n4, pp. 2173-2184, Nov. 2011.

[18]S. Al Aimani, "Modélisation de différentes technologies d'éoliennes intégrées à un réseau de distribution moyenne tension", PhD. Thesis, Ecole Centrale de Lille, chap. 2, pp. 24-25, 2004. 